\title{
Antihydrogen formation mechanisms
}

\author{
E. Lodi-Rizzini ${ }^{1,2, a}$, M. Charlton ${ }^{3}$, R.S. Hayano ${ }^{4}$, A. Rotondi ${ }^{5,6}$, L. Venturelli ${ }^{1,2}$, and N. Zurlo ${ }^{1,2}$ \\ ${ }^{1}$ Dipartimento di Ingegneria dell'Informazione, Università di Brescia, I-25123 Brescia, Italy \\ ${ }^{2}$ Istituto Nazionale di Fisica Nucleare, Gruppo Collegato di Brescia, I-25123 Brescia, Italy \\ ${ }^{3}$ Department of Physics, College of Science, Swansea University, Singleton Park, Swansea SA2 8PP, United \\ Kingdom \\ ${ }^{4}$ Department of Physics, University of Tokyo. Tokyo 113-0033, Japan \\ ${ }^{5}$ Dipartimento di Fisica Nucleare e Teorica, Università di Pavia, 27100 Pavia, Italy \\ ${ }^{6}$ Istituto Nazionale di Fisica Nucleare, Sezione di Pavia, 27100 Pavia, Italy
}

\begin{abstract}
New developments in the ATHENA data analysis are presented, with some insight into the mechanisms responsible for antihydrogen production under different experimental conditions.
\end{abstract}

Studies of antihydrogen, the bound state of a positron and an antiproton, can offer new insights into the fundamental symmetries of nature from comparisons of its properties with those of hydrogen. The production and trapping of antihydrogen at low energies has recently become possible, using specialised apparatus developed at CERN [1-5]. There has been no detailed study of the processes involved in the formation of antihydrogen via antiproton-positron plasma mixing in a Penning trap environment, and much has been assumed from equilibrium studies at higher plasma temperatures and in the absence of applied fields. It is the nature of the mechanisms underlying antihydrogen formation that we address in this work. In particular, we show that a careful analysis of the correlated temporal and spatial dependencies of the antihydrogen on wall annihilation signals, available from a range of experiments performed by the ATHENA collaboration, can shed light on this problem.

ATHENA aimed at mixing antiprotons (pbar) and positrons $\left(e^{+}\right)$and consisted of four main subsystems: the antiproton catching trap, the positron accumulator, the antiproton/positron mixing trap, and the antihydrogen annihilation detector (see Figure 1 and [6] for the datails). The positrons $\left(\sim 10^{8} e^{+}\right)$and antiprotons $\left(\sim 10^{4}\right.$ pbars) were held in devices known as nested Penning traps (see e.g. [7]) which provide confinement using a combination of applied fields. A series of cylindrical electrodes, aligned along the axis of a solenoid, were used to produce electrical wells which restrict the particles axially, while the $\sim 3 \mathrm{~T}$ magnetic field provides transverse confinement (see Figure 2).

When an antiproton and a positron, after interacting, are bound together, they form an antihydrogen atom (Hbar) which is neutral and which thus eventually impinges on the electrode walls and annihilates. The simultaneous annihilation of the pbar on a proton/neutron (producing charged pions) and of the $e^{+}$on an electron (producing two $\gamma$-rays) is detected by a cylindrical detector which is also able to pinpoint the annihilation vertex (see Figure 3).

Although ATHENA has produced over the years a large variety of interesting results ([6, 8-19]), here we are interested in some possible hints about the mechanism responsible for antihydrogen pro-

\footnotetext{
ae-mail: lodi@bs.infn.it
} 
duction which, under the conditions of the ATHENA experiment, is expected to be essentially of two kinds: spontaneous radiative recombination and 3-body recombination, see Figure 4 and e.g. [20].

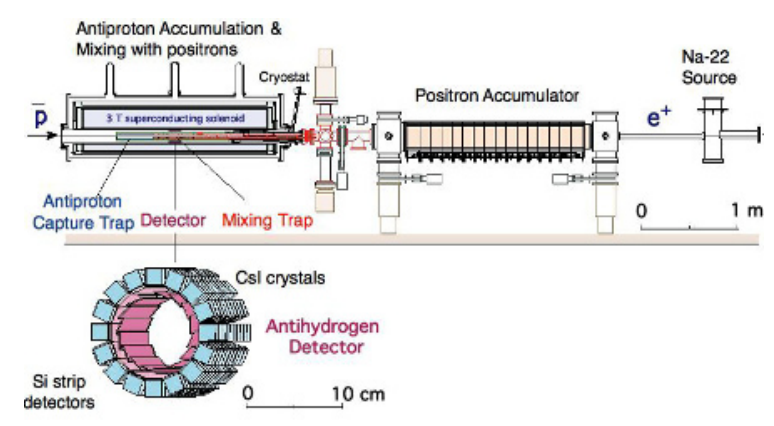

Figure 1. Overview of the ATHENA apparatus for the production and detection of antihydrogen. An expanded view of the annihilation detector is shown below the main apparatus.

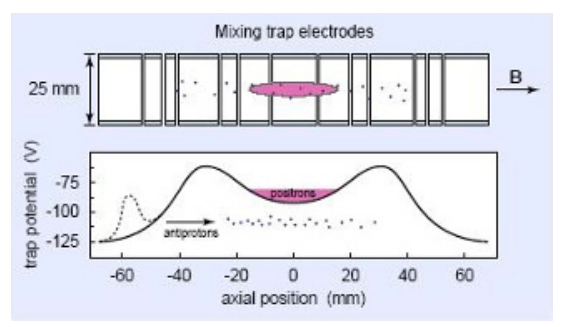

Figure 2. Scheme of the cylindrical electrodes constituting the nested Penning trap. The pink ellipse shows the positron plasma (with usual position and typical size), while the tiny crosses indicate the antiprotons. The same things are reported also in the lower panel, where the trapping electrostatic potential is plotted against the position along the trap axis. The dashed line is the potential before pbar injection, while the solid line is the potential during mixing.

For experiments where Hbars are produced by overlapping pbars and $e^{+}$, the Hbar production rate depends mainly on the $e^{+}$plasma temperature in both the expected Hbar formation mechanisms, with the dependence much stronger $\left(\propto T^{-4.5}\right)$ in the three-body recombination. In Figure 5, we can observe the on-wall antihydrogen annihilation time distribution in the standard situation (called "Cold Mixing"), showing a large peak at the beginning (i.e. at the injection, red fitting curve) followed after around $40 \mathrm{~s}$ by a change in the potential of the two lateral wells which produces a reinjection of the antiprotons in the positrons cloud (green fitting curve) [11]. From the ratio between the two peak heights, we can expect that around 70\%-80\% of the antihydrogen produced at the injection occurs via 3-body recombination with low binding energies, and after the consequent ionization near the edge of the positron cloud, the antiprotons are captured in the two lateral wells. When we perform such a reinjection, the Hbar production occurs in ATHENA apparatus with a rate $\left(\lambda_{2}\right)$ very similar to the rate after the injection $\left(\lambda_{1}\right)$. Moreover, these data support the idea that the positron cloud conditions after $40 \mathrm{~s}$ are the same as in the first seconds (because of the strong dependence on the temperature, a change of a few degrees in temperature would change the rate $\lambda$ by an order of magnitude).

It is important to stress that in Figure 5 and the subsequent Figure 6 what we call "annihilation temporal distributions" are the temporal distributions of the reconstructed annihilations vertices, which can be selected in order to avoid most of non-antihydrogen events (see e.g. [19]). 


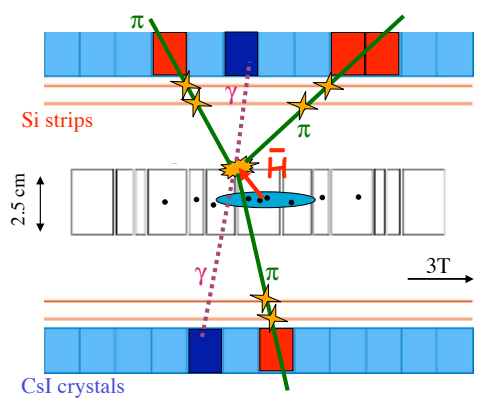

Figure 3. Schematic diagram of the ATHENA mixing trap and the surrounding antihydrogen detector. The cylindrical electrodes and the position of the positron cloud (blue ellipse) are shown. A typical antihydrogen annihilation is also shown: the green lines correspond to the escaping charged pions from pbar-nuclei annihilation, with the red dotted lines depicting the two back-to-back rays from $e^{+} e^{-}$annihilation.

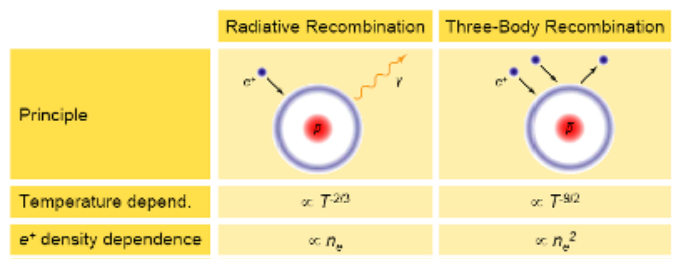

Figure 4. Sketch of the radiative recombination vs 3-body recombination, with their respective dependencies on the positron plasma temperature and density.

By performing measurements over a range of positron plasma densities it is possible to determine the scaling of the rate values $\left(\lambda_{1}\right.$ and $\lambda_{2}$, see Figure 6$)$ with the $e^{+}$cloud density. This analysis suggests that, in addition to the 3-body collisional process [9] (red line, scaling with the $e^{+}$density squared), for the first time there are hints for a contribution to antihydrogen formation from the 2-body radiative mechanism, since the antihydrogen detected at late times (blue line) scales linearly with the $e^{+}$density.

In conclusion, the mixing of antiprotons and positrons at the CERN AD resulted in a copious production of antihydrogen atoms in different experimental situations. Differently from the hydrogen formation in the early Universe, where the radiative capture of an electron by a proton was the main mechanism, in the different $\mathrm{AD}$ experiments the antihydrogen formation has occured following mainly the three-body process where two $e^{+}$interact with the pbar. The very different conditions (different densities and temperatures up to $10^{4} \mathrm{~K}$ ) experimented in the ATHENA apparatus have produced antihydrogen atoms possibly by means of the radiative capture too. Any information on the antihydrogen production mechanisms will be useful for the goal of precision spectroscopy of antihydrogen.

\section{References}

[1] M. Amoretti, et al., Nature 419, 456-459 (2002).

[2] G. Gabrielse, et al., Phys. Rev. Lett. 89, 213401 (2002).

[3] G.B. Andresen, et al., Nature 468 673-676 (2010).

[4] Y. Enomoto, et al., Phys. Rev. Lett. 105243401 (2010). 


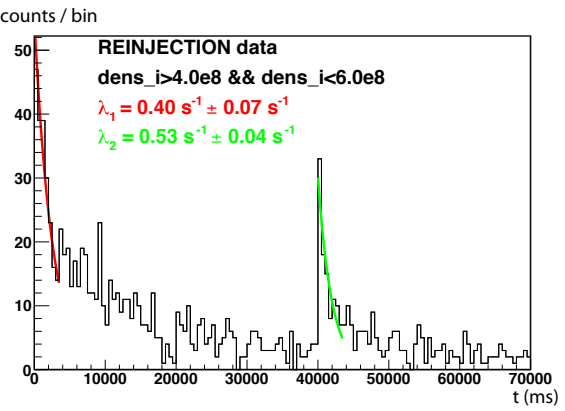

Figure 5. Annihilation temporal distributions for standard Cold Mixing followed by reinjection after $40 \mathrm{~s}$. The $e^{+}$plasma density is between $4 \times 10^{8} \mathrm{~cm}^{-3}$ and $6 \times 10^{8} \mathrm{~cm}^{-3}$. The parameters $\lambda_{1}$ and $\lambda_{2}$ are the inverse time constants determined by force-fitting the exponential curve $k e^{-\lambda t}$ ( $\lambda_{1}$ relates to the injection, $\lambda_{2}$ to the reinjection).
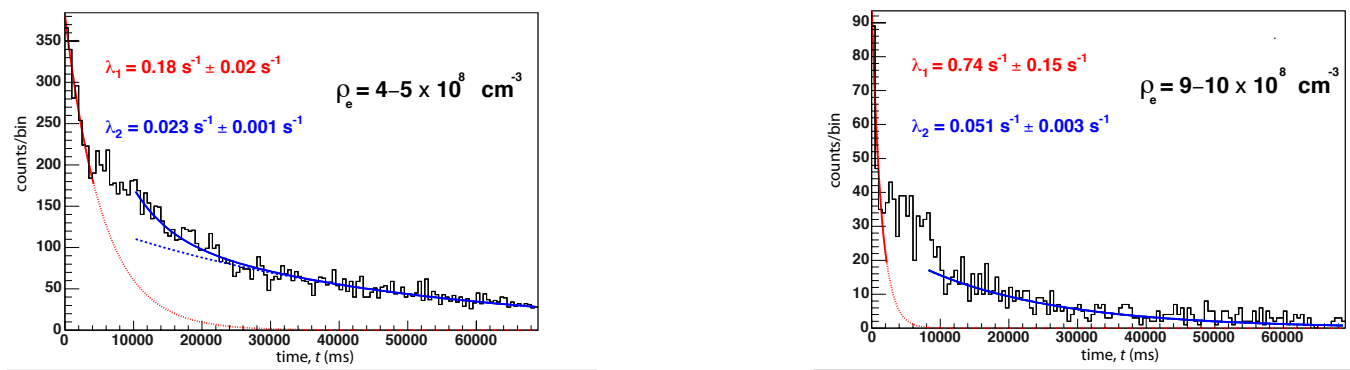

Figure 6. Annihilation temporal distributions for standard Cold Mixing at different densities: on the right, the data were taken with a density about twice than on the left. Here, $\lambda_{1}$ and $\lambda_{2}$ refer again to the result of force-fitting the exponential curve $k e^{-\lambda t}$, but here $\lambda_{1}$ refers to the first seconds, $\lambda_{2}$ refers to the last tens of seconds.

[5] G. Gabrielse, et al., Phys. Rev. Lett. 108, 113002 (2012).

[6] M. Amoretti, et al., Nuc. Instr. Meth. A 518, 679-711 (2004).

[7] F.G. Major, V.N. Gheorghe, and G. Werth, Charged particle traps (Springer, Berlin) (2005).

[8] M. Amoretti, et al., Phys. Rev. Lett. 91, 055001 (2003).

[9] M. Amoretti, et al., Phys. Letts B 578, 23-32 (2004).

[10] M. Amoretti, et al., Phys. Letts B 583, 59-67 (2004).

[11] M. Amoretti, et al., Phys. Letts B 590, 133-142 (2004).

[12] M.C. Fujiwara et al., Phys. Rev. Lett. 92, 065005 (2004).

[13] N. Madsen, et al., Phys. Rev. Lett. 94, 033403 (2005).

[14] L.V. Jorgensen, et al., Phys. Rev. Lett. 95, 025002 (2005).

[15] M. Amoretti, et al., Phys. Rev. Lett. 97, 213401 (2006).

[16] A. Kellerbauer, et al., Phys. Rev. A 73, 062508 (2006).

[17] R. Funakoshi, et al., Phys. Rev. A 76, 012713 (2007).

[18] M.C. Fujiwara et al., Phys. Rev. Lett. 101, 053401 (2008).

[19] N. Zurlo et al., Phys. Rev. Lett. 97, 153401 (2006).

[20] M.H. Holzscheiter, M. Charlton and M.M. Nieto, Phys. Rep. 402, 1-101 (2004). 